\title{
Weak Compactness and Weak Essential Spectra of Elementary Operators
}

\author{
EERO SAKSMAN
}

Abstract. Let $E$ be a Banach space and let $A=\left(A_{1}, \ldots, A_{n}\right)$ and $B=\left(B_{1}, \ldots, B_{n}\right)$ be $n$-tuples of operators on $E$. The elementary operator $\mathcal{E}_{A, B}: L(E) \rightarrow L(E)$ is defined by $\mathcal{E}_{A, B}=\sum_{i=1}^{n} L_{A_{i}} R_{B_{i}}$, where $L_{T}$ and $R_{T}$ denote the multiplication operators $L_{T} U=T U$ and $R_{T} U=U T$ for $U \in L(E)$. This paper studies weak compactness and spectra modulo $W(L(E)$ ) of elementary operators, and it extends the most important results obtained previously for single two-sided multiplication operators.

In the case that $E=\ell^{p}, 1<p<\infty$, we give a characterization for the weak compactness of $\mathcal{E}_{A, B}$ and show that $\sigma_{w}\left(\mathcal{E}_{A, B}\right)=$ $\sigma_{T e}(A) \circ \sigma_{T e}(B)$, where $\sigma_{T e}$ denotes the Taylor essential spectrum and $A$ and $B$ are commuting $n$-tuples of operators. Similarly, in the case that $E^{\prime}$ has the Dunford-Pettis property we characterize the weak compactness and show that $\sigma_{w}\left(\mathcal{E}_{A, B}\right)=\sigma_{e}\left(\mathcal{E}_{A, B}\right)=\sigma_{T e}(A) \circ$ $\sigma_{T}(B) \cup \sigma_{T}(A) \circ \sigma_{T e}(B)$, where $\sigma_{T}$ denotes the Taylor spectrum. The essential spectrum of $\mathcal{E}_{A, B}$ has been computed before by Curto, Fialkow and Eschmeier, and our techniques yield also a new proof of their result. Most of our results remain valid for restrictions of $\mathcal{E}_{A, B}$ to Banach ideals $I$ of $L(E)$.

A weakly compact analogue of the Stampfli identity for the norm of an inner derivation is established by computing the distance to $W\left(L\left(\ell^{2}\right)\right)$ of an inner derivation on $L\left(\ell^{2}\right)$. Finally, we determine the essential norm of a two-sided multiplication operator on $L\left(\ell^{2}\right)$ or on ideals $I$ of $L\left(\ell^{2}\right)$.

0. Introduction. Assume that $T \in L(E)$, where $E$ is a Banach space and $L(E)$ denotes the bounded linear operators on $E$. The left and right multiplica- 
tion operators $L_{T}, R_{T} \in L(L(E))$ are defined by $L_{T} X=T X$ and $R_{T} X=X T$ for $X \in L(E)$. More generally, if $A=\left(A_{1}, \ldots, A_{n}\right)$ and $B=\left(B_{1}, \ldots, B_{n}\right)$ are operator $n$-tuples on $E$ (that is, $A, B \in(L(E))^{n}$ ), the corresponding elementary operator $\mathcal{E}_{A, B}: L(E) \rightarrow L(E)$ is defined as

$$
\mathcal{E}_{A, B}=\sum_{i=1}^{n} L_{A_{i}} R_{B_{i}} .
$$

Elementary operators have been intensively studied during the last ten years: structural and spectral properties of $\mathcal{E}_{A, B}$ have been related to those of $A_{i}$ and $B_{i}$. There are several reasons for this. Elementary operators are closely related to tensor products of operators and they form an algebra which contains the elements of $L(L(E))$ of the simplest type, namely $L_{T}$ and $R_{T}$. Moreover, $\mathcal{E}_{A, B}$ preserves Banach ideals of $L(E)$ and, for $C^{*}$-algebras, this property characterizes the strong closure of elementary operators - see [25]. For accounts of previous results on structural properties, spectra and essential spectra of $\mathcal{E}_{A, B}$, we refer to the excellent surveys due to Curto [9] and Fialkow [15].

This paper studies the weak compactness and the weak essential spectra of elementary operators. The corresponding questions were considered previously by Ogasawara [27], Ylinen [37], Akemann and Wright [1] and Mathieu [26] for elementary operators on $C^{*}$-algebras, by Diestel and Faires [11] for tensor products of operators, and by Saksman and Tylli [30], [31] and Racher [28] for two-sided multiplication operators $L_{T} R_{S}$ on $L(E)$ with arbitrary Banach spaces $E$. We extend several of the most important results of these papers to elementary operators on $L(E)$ (or on Banach ideals of $L(E)$ ). The results of [30] suggest that (roughly speaking) definite results are to be expected if $E=\ell^{p}(1<p<\infty)$ or when $E$ has the Dunford-Pettis property (for example, the spaces $\ell^{1}, \ell^{\infty}, C(0,1)$, $\left.L^{1}(0,1)\right)$. We next indicate our main results.

In the case of $\ell^{p}$-spaces $(1<p<\infty)$, we obtain a qualitative generalization of [31, Theorem 2.i]: Suppose that $A$ and $B$ are $n$-tuples of operators on $\ell^{p}$ and assume that the operators $B_{1}, \ldots, B_{n}$ are linearly independent modulo $K\left(\ell^{p}\right)$, where $K\left(\ell^{p}\right)$ denotes the compact linear operators on $L\left(\ell^{p}\right)$. Then the operator $\mathcal{E}_{A, B}$ is weakly compact if and only if $A_{i} \in K\left(\ell^{p}\right)$ for all $i=1, \ldots, n$. Moreover, if $A$ and $B$ are commuting $n$-tuples of operators on $\ell^{p}$, it holds that (compare $[31$, Theorem 4(ii)])

$$
\sigma_{w}\left(\mathcal{E}_{A, B}\right)=\sigma_{T e}(A) \circ \sigma_{T e}(B)=\sigma_{H e}(A) \circ \sigma_{H e}(B),
$$

where $\sigma_{w}$ denotes the weak essential spectrum, that is, the quotient spectrum in the weak Calkin algebra $L\left(L\left(\ell^{p}\right)\right) / W\left(L\left(\ell^{p}\right)\right.$ ), and the indexes $T$ (resp. $H$ ) refer to Taylor's (resp. Harte's) joint spectrum (see Section 1 for notations). These results remain valid also if $\mathcal{E}_{A, B}$ acts on a non-reflexive Banach ideal of $L\left(\ell^{p}\right)$.

In the case that $E$ has the Dunford-Pettis property, Racher's characterization [28, Proposition 2] generalizes as follows: The elementary operator $\mathcal{E}_{A, B}$ : 
$L(E) \rightarrow L(E)$ is weakly compact if and only if it may be written in the form $\mathcal{E}_{A, B}=\mathcal{E}_{U, V}$, where each component of the operator tuples $U$ and $V$ is weakly compact. We provide a weakly compact counterpart of the basic results due to Fialkow [14], Carrillo and Hernandez [4], Curto and Fialkow [10], and Eschmeier [12] concerning the essential spectrum of $\mathcal{E}_{A, B}$. It turns out that

$$
\begin{aligned}
\sigma_{w}\left(\mathcal{E}_{A, B}\right)=\sigma_{e}\left(\mathcal{E}_{A, B}\right) & =\sigma_{T e}(A) \circ \sigma_{T}(B) \cup \sigma_{T}(A) \circ \sigma_{T e}(B) \\
& =\sigma_{H e}(A) \circ \sigma_{H}(B) \cup \sigma_{H}(A) \circ \sigma_{H e}(B)
\end{aligned}
$$

for commuting operator $n$-tuples $A$ and $B$ under the assumption that $E^{\prime}$ has the Dunford-Pettis property. This result holds also for elementary operators on Banach ideals of $L(E)$. We point out that our techniques yield a conceptually simpler proof of the Fialkow-Eschmeier formula for $\sigma_{e}\left(\mathcal{E}_{A, B}\right)$, valid for all Banach spaces.

Let $A, B \in L\left(\ell^{2}\right)$. It is well known that all derivations on $L\left(\ell^{2}\right)$ are inner, i.e. of form $D_{A}:=L_{A}-R_{A}$ for some $A \in L\left(\ell^{2}\right)$. In Section 3 we compute the distance of derivations to the weakly compact operators:

$$
\left\|D_{A}: L\left(\ell^{2}\right) \rightarrow L\left(\ell^{2}\right)\right\|_{w}=2 \inf _{\lambda \in \mathbb{C}}\|A-\lambda\|_{e} .
$$

In addition, we establish the formula

$$
\left\|L_{A} R_{B}: L\left(\ell^{2}\right) \rightarrow L\left(\ell^{2}\right)\right\|_{e}=\max \left\{\|A\|_{e}\|B\|,\|A\|\|B\|_{e}\right\},
$$

which remains true also if $L_{A} R_{B}$ acts on a Banach ideal of $L\left(\ell^{2}\right)$. These results complement the previous computations of various norms due to Stampfli [33, Theorem 4], Fong [16], Sun [34], and Saksman and Tylli [30, Theorem 2(ii)].

We express our gratitude to H.-O. Tylli for suggesting some of the problems considered here and for his continuous interest in our work.

1. Preliminaries. We shall use the standard notations of Banach space theory. Especially, if $E$ is a Banach space, $B_{E}$ denotes the unit ball of $E, L(E)$ (resp. $K(E), W(E)$ ) denotes the bounded (resp. compact, weakly compact) linear operators on $E$. In this paper all Banach spaces are assumed to be complex. The norm of an operator $T \in L(E)$ in the Calkin algebra $\mathcal{C}(E)=L(E) / K(E)$ is denoted by $\|T\|_{e}:=\|T+K(E)\|_{\mathcal{C}(E)}=\operatorname{dist}(T, K(E))$. For non-reflexive spaces $E$ the weak Calkin algebra is $\mathcal{W}(E)=L(E) / W(E)$, and the weak essential norm is defined as $\|T\|_{w}:=\|T+W(E)\|_{\mathcal{W}(E)}=\operatorname{dist}(T, W(E))$. The essential spectrum $\sigma_{e}(T)$ is the set of $\lambda \in \mathbb{C}$ such that $T-\lambda$ fails to be Fredholm. It is well known that this is equivalent to $T-\lambda$ not being invertible modulo the compact operators so that $\sigma_{e}(T)=\sigma(T+K(E))$. Analogously, the weak essential spectrum of $T$ is defined through $\sigma_{w}(T):=\sigma(T+W(E))$, and we call $T$ weak Fredholm if it is invertible modulo the weakly compact operators, that is, $0 \notin \sigma_{w}(T)$.

Consider a complex Banach algebra $\mathcal{A}$ with identity $1_{\mathcal{A}}$. Let $a=$ $\left(a_{1}, \ldots, a_{n}\right) \in \mathcal{A}^{n}$. We say that $a$ is a commuting $n$-tuple if $a_{i} a_{j}=a_{j} a_{i}$ 
for all $i, j \in\{1, \ldots, n\}$. The product $a \circ b$ of $n$-tuples $a$ and $b$ is defined as $a \circ b:=\sum_{i=1}^{n} a_{i} b_{i}$ and, accordingly, $G_{1} \circ G_{2}:=\left\{\sum_{i=1}^{n} \lambda_{i} \mu_{i} \mid \lambda \in G_{1}, \mu \in G_{2}\right\}$ for subsets $G_{1}, G_{2} \subset \mathbb{C}^{n}$. In what follows we recall the joint spectra that will be used later. For general properties of various joint spectra we refer to [8] - see also [35], [18], and [32]. If $a \in \mathcal{A}^{n}$ is a commuting $n$-tuple, one sets

$$
\begin{aligned}
\sigma_{\ell}(a) & :=\left\{\lambda \in \mathbb{C}^{n} \mid b \circ(a-\lambda) \neq 1_{\mathcal{A}} \text { for all } b \in \mathcal{A}^{n}\right\} \quad \text { (the left spectrum), } \\
\sigma_{r}(a) & :=\left\{\lambda \in \mathbb{C}^{n} \mid(a-\lambda) \circ b \neq 1_{\mathcal{A}} \text { for all } b \in \mathcal{A}^{n}\right\} \quad \text { (the right spectrum), } \\
\sigma_{H}(a) & :=\sigma_{\ell}(a) \cup \sigma_{r}(a) \quad \text { (the Harte spectrum), }
\end{aligned}
$$

and letting $a^{\prime \prime}$ denote the bicommutant of the commuting set $\left\{a_{1}, \ldots, a_{n}\right\}$, the (joint) bicommutant spectrum of $a$ is

$$
\sigma^{\prime \prime}(A):=\left\{\lambda \in \mathbb{C}^{n} \mid(a-\lambda) \circ b=b \circ(a-\lambda) \neq 1_{\mathcal{A}} \text { for all } b \in\left(a^{\prime \prime}\right)^{n}\right\} .
$$

Next we specialize in operator algebras and their quotient algebras. Assume that $E$ is a (complex) Banach space and let $A=\left(A_{1}, \ldots, A_{n}\right)$ stand for a commuting $n$-tuple of operators on $E\left(A_{i} \in L(E)\right.$ for all $\left.i=1, \ldots, n\right)$. The spectra $\sigma_{\ell}(A), \sigma_{r}(A)$ and $\sigma_{H}(A)$ are defined as before with respect to the algebra $L(E)$. In addition we define $\sigma_{H e}(A)=\sigma_{H}\left(A_{1}+K(E), \ldots, A_{n}+K(E)\right)$ with respect to the algebra $\mathcal{C}(E)$ and $\sigma_{H w}(A)=\sigma_{H}\left(A_{1}+W(E), \ldots, A_{n}+W(E)\right)$ with respect to the algebra $\mathcal{W}(E)$. The definitions of $\sigma_{\ell e}(A), \sigma_{r e}(A), \sigma_{\ell w}(A)$ and $\sigma_{r w}(A)$ are analogous.

The (joint) approximate point spectrum $\sigma_{\pi}$ and the (joint) approximate defect spectrum $\sigma_{\delta}$ are defined as

$$
\sigma_{\pi}(A)=\left\{\lambda \in \mathbb{C}^{n} \mid \inf _{x \in E,\|x\|=1} \sum_{i=1}^{n}\left\|\left(A_{i}-\lambda_{i}\right) x\right\|=0\right\}
$$

and $\sigma_{\delta}(A)=\sigma_{\pi}\left(A^{\prime}\right)$, where $A^{\prime}=\left(A_{1}^{\prime}, \ldots, A_{n}^{\prime}\right)$ (cf. [32, Lemma 2.10]). We denote $\sigma_{a}(A)=\sigma_{\pi}(A) \cup \sigma_{\delta}(A)$. For the purposes of the present work a special concept is needed: We define $\sigma_{\pi e}$ and $\sigma_{\delta e}$ by

$$
\begin{aligned}
\sigma_{\pi e}(A) & =\sigma_{\pi}\left(L_{A_{1}+K(E)}, \ldots, L_{A_{n}+K(E)}\right), \\
\sigma_{\delta e}(A) & =\sigma_{\pi}\left(R_{A_{1}+K(E)}, \ldots, R_{A_{n}+K(E)}\right) .
\end{aligned}
$$

In the definition above, the multiplication operators act on the Calkin algebra $\mathcal{C}(E)$. In addition, we denote $\sigma_{a e}(A)=\sigma_{\pi e}(A) \cup \sigma_{\delta e}(A)$. The definition of $\sigma_{a e}$ implies that $\lambda \in \sigma_{a e}(A)$ if and only if there is a sequence $\left(T_{k}\right)_{k=1}^{\infty}$ in $L(E)$ with $\left\|T_{k}\right\|_{e}=1$ for all $k$ and $\sum_{i=1}^{n}\left\|T_{k}\left(A_{i}-\lambda_{i}\right)\right\|_{e} \rightarrow 0$ or $\sum_{i=1}^{n}\left\|\left(A_{i}-\lambda_{i}\right) T_{k}\right\|_{e} \rightarrow 0$ as $k \rightarrow \infty$.

Finally, the Taylor spectrum $\sigma_{T}(A)$ (resp. the Taylor essential spectrum $\left.\sigma_{T e}(A)\right)$ of the operator $n$-tuple $A$ is defined as the set of $\lambda \in \mathbb{C}^{n}$ such that the Koszul complex corresponding to $A-\lambda$ fails to be exact (resp. Fredholm). 
Since our results involving the Taylor spectra are direct consequences of Proposition 1 below, we omit the detailed definition and refer to [35] and [8] for this concept.

All the joint spectra $\sigma_{H}, \sigma_{H e}, \sigma_{H w}, \sigma_{a}, \sigma_{a e}, \sigma_{T}$ and $\sigma_{T e}$ above obey the (scalar) polynomial spectral mapping theorem stating that, for polynomials $P$ : $\mathbb{C}^{n} \rightarrow \mathbb{C}$, it holds that $\sigma_{\{\cdot\}}(P(A))=P\left(\sigma_{\{\cdot\}}(A)\right)$ - see [18], [35], and [32]. The fact that the spectral mapping theorem holds also for the spectrum $\sigma_{a e}$ follows by noting that $\sigma_{\pi e}$ and $\sigma_{\delta e}$ are the algebraic approximate point and defect spectra in the Calkin algebra $\mathcal{C}(E)$ (the spectral mapping theorem holds also for algebraic approximate spectra; see [32, p. 136] or [9, p. 42]).

The double commutant spectrum $\sigma^{\prime \prime}$ also satisfies the scalar polynomial spectral mapping theorem, although $\sigma^{\prime \prime}$ does not even have the projection property (see [32, pp. 144-145]). Namely, for commuting $n$-tuples $a$ in a Banach algebra $\mathcal{A}$, the definitions yield that $\sigma^{\prime \prime}(a)=\sigma_{H}^{a^{\prime \prime}}(a)$, where $\sigma_{H}^{a^{\prime \prime}}$ denotes the Harte spectrum with respect to the bicommutant subalgebra $a^{\prime \prime}$ of $\mathcal{A}$. Applying the spectral mapping theorem for $\sigma_{H}$ in $a^{\prime \prime}$ with any (scalar) polynomial $P: \mathbb{C}^{n} \rightarrow \mathbb{C}$, we deduce that $P\left(\sigma^{\prime \prime}(a)\right)=P\left(\sigma_{H}^{a^{\prime \prime}}(a)\right)=\sigma^{a^{\prime \prime}}(P(a))=\sigma(P(a))$, where the last equality is a well-known property of bicommutant spectra.

Next we record a useful observation, the idea of which is due to Curto [7, Theorem 3.15], but we include the proof for completeness.

Proposition 1. Let $E$ be a Banach space and let $A$ and $B$ be commuting $n$-tuples of operators on $E$. Assume that $P: \mathbb{C}^{2 n} \rightarrow \mathbb{C}$ is a polynomial. Then

$$
\begin{aligned}
P\left(\sigma_{T e}(A) \times \sigma_{T}(B)\right) & =P\left(\sigma_{H e}(A) \times \sigma_{H}(B)\right)=P\left(\sigma_{H e}(A) \times \sigma_{a}(B)\right) \\
& =P\left(\sigma_{H e}\left(A^{\prime}\right) \times \sigma_{a}(B)\right)
\end{aligned}
$$

and, in the case of $E=\ell^{p}$ with $1<p<\infty$, it also holds that

$$
P\left(\sigma_{T e}(A) \times \sigma_{T e}(B)\right)=P\left(\sigma_{H e}(A) \times \sigma_{H e}(B)\right)=P\left(\sigma_{a e}(A) \times \sigma_{a e}(B)\right) .
$$

Proof. We first prove the inclusion $P\left(\sigma_{H e}(A) \times \sigma_{H}(B)\right) \subset P\left(\sigma_{H e}\left(A^{\prime}\right) \times\right.$ $\left.\sigma_{a}(B)\right)$. Assume hence that $\left(\lambda_{0}, \mu_{0}\right) \in \sigma_{H e}(A) \times \sigma_{H}(B)$, and consider the polynomial $Q_{1}$ of $n$ variables, where $Q_{1}(z)=P\left(z, \mu_{0}\right)$ for $z \in \mathbb{C}^{n}$. By applying the spectral mapping theorem and the well-known equality $\sigma_{e}(T)=\sigma_{e}\left(T^{\prime}\right)$ for single operators $T \in L(E)$, we obtain

$$
Q_{1}\left(\sigma_{H e}(A)\right)=\sigma_{e}\left(Q_{1}(A)\right)=\sigma_{e}\left(\left(Q_{1}(A)\right)^{\prime}\right)=\sigma_{e}\left(Q_{1}\left(A^{\prime}\right)\right)=Q_{1}\left(\sigma_{H e}\left(A^{\prime}\right)\right) .
$$

Hence $P\left(\sigma_{H e}(A), \mu_{0}\right)=P\left(\sigma_{H e}\left(A^{\prime}\right), \mu_{0}\right)$, which implies that there is $\lambda_{1} \in \sigma_{H e}\left(A^{\prime}\right)$ such that $P\left(\lambda_{0}, \mu_{0}\right)=P\left(\lambda_{1}, \mu_{0}\right)$. In a similar manner, by considering the polynomial $Q_{2}$ of $n$ variables, where $Q_{2}(z)=P\left(\lambda_{1}, z\right)$ for $z \in \mathbb{C}^{n}$, we see that $Q_{2}\left(\sigma_{H}(B)\right)=Q_{2}\left(\sigma_{a}(B)\right)$, and thus there is $\mu_{1} \in \sigma_{a}(B)$ with the property $P\left(\lambda_{1}, \mu_{0}\right)=P\left(\lambda_{1}, \mu_{1}\right)$. Hence $P\left(\lambda_{0}, \mu_{0}\right)=P\left(\lambda_{1}, \mu_{0}\right)=P\left(\lambda_{1}, \mu_{1}\right)$, where $\left(\lambda_{1}, \mu_{1}\right)$ $\in \sigma_{H e}\left(A^{\prime}\right) \times \sigma_{a}(B)$, and the desired inclusion follows. The proof of (1.1) is 
completed by noting that the reverse inclusion is proved in a completely analogous manner and that the proof of the other equalities is similar. The same argument yields (1.2) once we recall that $\sigma_{e}(T)=\sigma_{a e}(T)$ for single operators $T \in L\left(\ell^{p}\right)$. Indeed, it is evident that $\sigma_{a e}(T) \subset \sigma_{e}(T)$ and the other direction follows from [21, Corollary 6.11] since for $1<p<\infty$ the spaces $\ell^{p}$ are both suband superprojective.

A Banach ideal $I$ of $L(E)$ is a two-sided ideal of $L(E)$ equipped with a complete norm $\|\cdot\|_{I}$ such that

(1) $\left\|x^{\prime} \otimes y\right\|_{I}=\left\|x^{\prime}\right\|\|y\|$ for $x^{\prime} \in E^{\prime}, y \in E$;

(2) $\|S\| \leq\|S\|_{I}$ and

(3) $\|U S V\|_{I} \leq\|U\|\|V\|\|S\|_{I}$ for $U, V \in L(E)$ and $S \in I$.

Assume that $T$ is an $n$-tuple of left or right multiplication operators or that $T$ is an elementary operator. If needed, we use notations like $\sigma_{T e}(T: I \rightarrow I), \sigma_{w}(T$ : $I \rightarrow I), \ldots$ to indicate that $T$ is considered on $I$.

Finally, we recall the Dunford-Pettis property (DPP for short): the space $E$ is said to have the DPP if for all weakly null sequences $\left(x_{k}^{\prime}\right)$ in $E^{\prime}$ and $\left(x_{k}\right)$ in $E$ it holds that $\lim _{k \rightarrow \infty}\left\langle x_{k}^{\prime}, x_{k}\right\rangle=0$. An important consequence of this property is that $T S \in K(E)$ for all $T, S \in W(E)$.

2. Weak compactness of elementary operators. This section studies the weak compactness of elementary operators. Let $E$ be a Banach space and assume that $A$ and $B$ are (not necessarily commuting) $n$-tuples of bounded linear operators on $E$. It is useful to notice that if the elementary operator $\mathcal{E}_{A, B}$ is expressed in the form $\sum_{i=1}^{n} L_{A_{i}} R_{B_{i}}$ with a minimal number of terms, then the elements of the set $\left\{A_{1}, \ldots, A_{n}\right\}$ are linearly independent and the same holds for $\left\{B_{1}, \ldots, B_{n}\right\}$ - otherwise the number of terms could be reduced.

We begin with a necessary condition for weak compactness.

Proposition 2. Let $E$ be a Banach space and assume that $A$ and $B$ are $n$-tuples of operators on $E$ such that the elementary operator $\mathcal{E}_{A, B}$ is expressed with a minimal number of terms as $\mathcal{E}_{A, B}=\sum_{i=1}^{n} L_{A_{i}} R_{B_{i}}$. Let $I$ be a Banach ideal of $L(E)$. Then the condition $\mathcal{E}_{A, B} \in W(I)$ implies that $A_{i} \in W(E)$ and $B_{i} \in W(E)$ for all $i=1, \ldots, n$.

Proof. Assume that $\mathcal{E}_{A, B}$ is weakly compact. By the assumed minimality of the representation, the sets $\left\{A_{1}, \ldots, A_{n}\right\}$ and $\left\{B_{1}, \ldots, B_{n}\right\}$ are linearly independent. According to [17, Lemma 1] there are $m \in \mathbb{N}, x_{1}, \ldots, x_{m}$ in $E$ and $x_{1}^{\prime}, \ldots, x_{m}^{\prime}$ in $E^{\prime}$ with the property $\sum_{k=1}^{m}\left\langle x_{k}^{\prime}, B_{j} x_{k}\right\rangle=\delta_{1, j}$ for $j=1, \ldots, n$, where $\delta$ denotes the Kronecker delta. It follows that $A_{1} x=\sum_{k=1}^{m}\left(\mathcal{E}_{A, B}\left(x_{k}^{\prime} \otimes x\right)\right) x_{k}$ for $x \in E$, which implies that $A_{1}$ is weakly compact as a product of bounded operators, one of which is weakly compact (note that the canonical imbedding 
$I \subset L(E)$ is bounded). The proof of $A_{2}, \ldots, A_{n} \in W(E)$ is similar. Next we apply the same lemma in order to find $y_{1}, \ldots, y_{l}$ in $E$ and $y_{1}^{\prime}, \ldots, y_{l}^{\prime}$ in $E^{\prime}$ such that $\sum_{k=1}^{\ell}\left\langle y_{k}^{\prime}, A_{j} y_{k}\right\rangle=\delta_{1, j}$ for $j=1, \ldots, n$. We obtain for arbitrary $x \in E$ and $x^{\prime} \in E^{\prime}$ that

$$
\sum_{k=1}^{\ell}\left\langle y_{k}^{\prime},\left(\mathcal{E}_{A, B}\left(x^{\prime} \otimes y_{k}\right)\right) x\right\rangle=\sum_{i=1}^{n} \sum_{k=1}^{\ell}\left\langle y_{k}^{\prime}, A_{i} y_{k}\right\rangle\left\langle B_{i}^{\prime} x^{\prime}, x\right\rangle=\left\langle B_{1}^{\prime} x^{\prime}, x\right\rangle .
$$

Thus, $B_{1}^{\prime} x^{\prime}=\sum_{k=1}^{\ell}\left(\mathcal{E}_{A, B}\left(x^{\prime} \otimes y_{k}\right)\right)^{\prime} y_{k}^{\prime}$ for $x^{\prime} \in E^{\prime}$. It follows that $B_{1}^{\prime} \in W\left(E^{\prime}\right)$ since we may factorize $B_{1}^{\prime}: E^{\prime} \rightarrow I \rightarrow I^{\ell} \rightarrow(L(E))^{\ell} \rightarrow\left(L\left(E^{\prime}\right)\right)^{\ell} \rightarrow E^{\prime}$ through bounded linear operators, one of which is weakly compact. Hence also $B_{1} \in$ $W(E)$. The reasoning is similar for $B_{2}, \ldots, B_{n}$.

Next an exact criterion for the weak compactness of $\mathcal{E}_{A, B}: I \rightarrow I$ is established in the case where $E=\ell^{p}$ with $1<p<\infty$ and $I$ is a non-reflexive Banach ideal of $L\left(\ell^{p}\right)$. This extends the qualitative part of [30, Theorem 4(i)] and complements the results of Mathieu [26, Proposition 3.2 and Theorem 3.7], where the question of the weak compactness of elementary operators was settled in the framework of $C^{*}$-algebras (see also [1], [37]). It is perhaps of interest to notice that in the case of $I=L\left(\ell^{2}\right)$, our result follows by combining the Fong-Sourour range-inclusion result [17, Theorem 2] with Proposition 5 below. However, it is not known whether range inclusion type conditions also characterize the weak compactness of restrictions of $\mathcal{E}_{A, B}$ on Banach ideals, and [17, Theorem 2] deals only with the Hilbert space case. Instead, we shall apply an effective algebraic generalization of the Fong-Sourour result due to Magajna [24].

Theorem 3. Let $1<p<\infty$ and suppose that $I$ is a non-reflexive Banach ideal of $L\left(\ell^{p}\right)$. Assume that $A$ and $B$ are $n$-tuples of operators on $\ell^{p}$. Then the elementary operator $\mathcal{E}_{A, B}: I \rightarrow I$ is weakly compact if and only if there are operator $m$-tuples $U=\left(U_{1}, \ldots, U_{m}\right)$ and $V=\left(V_{1}, \ldots, V_{m}\right)$ on $\ell^{p}$ such that

$$
\mathcal{E}_{A, B}=\mathcal{E}_{U, V}
$$

and $U_{k} \in K\left(\ell^{p}\right)$ or $V_{k} \in K\left(\ell^{p}\right)$ for each $k \in\{1, \ldots, m\}$. In particular, if the operators $A_{i}, i=1, \ldots n$, are linearly independent $\bmod K\left(\ell^{p}\right)$, then $\mathcal{E}_{A, B}$ is weakly compact if and only if $B_{i} \in K\left(\ell^{p}\right)$ for all $i=1, \ldots n$.

Proof. The 'if' part follows directly from [31, Theorem 4(i)], which states that a two-sided multiplication operator $L_{T} R_{S}: I \rightarrow I$ is weakly compact if (and only if ) $T \in K\left(\ell^{p}\right)$ or $S \in K\left(\ell^{p}\right)$. In order to prove the 'only if' part, we first assume that the operators $A_{i}$ are linearly independent $\bmod K\left(\ell^{p}\right)$ and that $\mathcal{E}_{A, B}$ is weakly compact. Magajna's surprising result [24, Corollary 1] applies to the algebra $L\left(\ell^{p}\right)$ (this is mentioned in [24, p. 201]) and, as in the proof of [24, Corollary 2], it follows that there is a positive integer $r$ and operators 
$X_{j}, Y_{j} \in L\left(\ell^{p}\right), j=1, \ldots, r$, with the property $\sum_{j=1}^{r} X_{j} A_{i} Y_{j}=\delta_{i, 1} \operatorname{Id}_{\ell^{p}}$ for $i=1, \ldots, n$. A substitution gives

$$
R_{B_{1}}=\sum_{j=1}^{r} L_{X_{j}} \mathcal{E}_{A, B} L_{Y_{j}}
$$

Thus $R_{B_{1}} \in W(I)$ and [31, Theorem 4.i] implies that $B_{1} \in K\left(\ell^{p}\right)$. Similarly, one proves that $B_{i} \in K\left(\ell^{p}\right)$ for $i=2, \ldots, n$.

In order to establish the 'only if' part for the other stated condition one notes that we may assume that the operators $A_{1}, \ldots, A_{k}(k \leq n)$ are linearly independent $\bmod K\left(\ell^{p}\right)$ and that $A_{k+1}, \ldots, A_{n}$ are linear combinations of the previous ones mod $K\left(\ell^{p}\right)$. Substituting the corresponding linear combinations in the sum defining $\mathcal{E}_{A, B}$ and applying the already proven criteria, we obtain the desired expression for $\mathcal{E}_{A, B}$.

The following theorem generalizes Racher's result [28, Proposition 2].

Theorem 4. Let the Banach space $E$ have the DPP and assume that $A$ and $B$ are $n$-tuples of operators on $E$. Then the elementary operator $\mathcal{E}_{A, B}$ : $L(E) \rightarrow L(E)$ is weakly compact if and only if there are operator $m$-tuples $U=$ $\left(U_{1}, \ldots, U_{m}\right)$ and $V=\left(V_{1}, \ldots, V_{m}\right)$ on $E$ such that

$$
\mathcal{E}_{A, B}=\mathcal{E}_{U, V}
$$

and $U_{k}, V_{k} \in W(E)$ for each $k \in\{1, \ldots, m\}$.

Proof. The 'if' part is immediate from [28, Proposition 2] and the other direction follows directly from Proposition 2.

Remark. It is unknown to us whether Theorem 4 holds also for elementary operators on Banach ideals $I$ of $L(E)$ if $E$ has the DPP.

Finally we record a partial counterpart of the range-inclusion criterion [30, Theorem 2.3] - the proof goes unchanged for elementary operators. We refer to the monograph [23, Section 2, 1.e] for the concept of the approximation property.

Proposition 5. Let $E$ be a reflexive Banach space with the approximation property. Assume that $A$ and $B$ are $n$-tuples of operators on $E$. Then the elementary operator $\mathcal{E}_{A, B}: L(E) \rightarrow L(E)$ is weakly compact if and only if

$$
\mathcal{E}_{A, B} L(E) \subset K(E) .
$$


3. Weak essential spectra of elementary operators. In this section we determine the weak essential spectrum of the elementary operator $\mathcal{E}_{A, B}$, where $A, B$ are commuting $n$-tuples of operators on $\ell^{p}(1<p<\infty)$ or on spaces $E$ such that $E^{\prime}$ has the Dunford-Pettis property. Our results (Theorems 8 and 11 below) extend [31, Theorem 4(ii), Corollary 6(i)] and provide counterparts of the basic results due to Eschmeier [12, Corollary 3.3]:

$$
\begin{aligned}
\sigma\left(\mathcal{E}_{A, B}: I \rightarrow I\right) & =\sigma_{T}(A) \circ \sigma_{T}(B) \\
\sigma_{e}\left(\mathcal{E}_{A, B}: I \rightarrow I\right) & =\sigma_{T e}(A) \circ \sigma_{T}(B) \cup \sigma_{T}(A) \circ \sigma_{T e}(B) .
\end{aligned}
$$

These formulae hold for commuting $n$-tuples of operators $A, B$ on arbitrary Banach spaces $E$ and Banach ideals $I$ of $L(E)$.

For operator $n$-tuples $A=\left(A_{1}, \ldots, A_{n}\right)$ we use the standard notation $L_{A}=$ $\left(L_{A_{1}}, \ldots, L_{A_{n}}\right)$. Moreover, $\left(L_{A}, R_{B}\right)$ denotes the $2 n$-tuple $\left(L_{A_{1}}, \ldots, L_{A_{n}}, R_{B_{1}}\right.$, $\left.\ldots, R_{B_{n}}\right)$ and products of type $\left(L_{A}, R_{B}\right) \circ\left(L_{C}, R_{D}\right)$ are interpreted as $L_{A} \circ L_{C}+$ $R_{B} \circ R_{D}$. In general, it is not reasonable to expect that one could compute joint weak essential spectra of $2 n$-tuples $\left(L_{A}, R_{B}\right)=\left(L_{A_{1}}, \ldots, L_{A_{n}}, R_{B_{1}}, \ldots, R_{B_{n}}\right)$ in the manner Curto and Fialkow [10] did for the joint essential spectra in the case of $E=\ell^{2}$, since already the results of Eschmeier [12] contain only upper and lower estimates for $\sigma_{T}\left(L_{A}, R_{B}\right)$ and $\sigma_{T e}\left(L_{A}, R_{B}\right)$. Moreover, it appears hard to find a natural candidate for a 'Taylor weak essential spectrum' $\sigma_{T w}$, since probably there are no 'geometric' characterizations for weak Fredholm operators (only the Taylor split spectrum generalizes in a natural way, see $[9$, p. 31]). However, it is possible to determine $\sigma_{H w}\left(L_{A}, R_{B}\right)$ in the Hilbert space case.

Theorem 6. Let $A$ and $B$ be commuting operator $n$-tuples of operators on $\ell^{2}$ and let $I$ stand for a non-reflexive Banach ideal of $L\left(\ell^{2}\right)$. Then

$$
\begin{aligned}
\sigma_{\ell w}\left(\left(L_{A}, R_{B}\right): I \rightarrow I\right) & =\sigma_{\ell e}(A) \times \sigma_{r e}(B), \\
\sigma_{r w}\left(\left(L_{A}, R_{B}\right): I \rightarrow I\right) & =\sigma_{r e}(A) \times \sigma_{\ell e}(B) \text { and } \\
\sigma_{H w}\left(\left(L_{A}, R_{B}\right): I \rightarrow I\right) & =\sigma_{\ell e}(A) \times \sigma_{r e}(B) \cup \sigma_{r e}(A) \times \sigma_{\ell e}(B) .
\end{aligned}
$$

Proof. We first prove the inclusion

$$
\sigma_{\ell w}\left(\left(L_{A}, R_{B}\right): I \rightarrow I\right) \subset \sigma_{\ell e}(A) \times \sigma_{r e}(B) .
$$

Assume thus that $(\lambda, \mu) \notin \sigma_{\ell e}(A) \times \sigma_{r e}(B)$, where $\lambda, \mu \in \mathbb{C}^{n}$. In the case $\lambda \notin \sigma_{\ell e}(A)$ there is an operator $n$-tuple $S$ on $\ell^{2}$ and an operator $K \in K\left(\ell^{2}\right)$ such that $S \circ(A-\lambda)-K=\operatorname{Id}_{\ell^{2}}$. This implies that

$$
\left(L_{S}, 0\right) \circ\left(L_{A}-\lambda, R_{B}-\mu\right)=\operatorname{Id}_{I}+L_{K},
$$

where $L_{K} \in W(I)$ by Theorem 3 and hence $(\lambda, \mu) \notin \sigma_{\ell w}\left(L_{A}, R_{B}\right)$. The reasoning is analogous in the case that $\mu \notin \sigma_{r e}(B)$ and we obtain (3.4). The inclusion

$$
\sigma_{r w}\left(\left(L_{A}, R_{B}\right): I \rightarrow I\right) \subset \sigma_{r e}(A) \times \sigma_{\ell e}(B)
$$


is proved similarly.

Next we establish the converse inclusion $\sigma_{\ell w}\left(\left(L_{A}, R_{B}\right): I \rightarrow I\right) \supset \sigma_{\ell e}(A) \times$ $\sigma_{r e}(B)$. Suppose hence that $\lambda \in \sigma_{\ell e}(A)$ and $\mu \in \sigma_{r e}(B)$. Let $\left(\ell^{2}\right)^{n}=$ $\left(\ell^{2} \oplus \cdots \oplus \ell^{2}\right)_{\ell^{2}}$ with $n$ summands, and define $T \in L\left(\ell^{2},\left(\ell^{2}\right)^{n}\right)$ by

$$
T x=\left(\left(A_{1}-\lambda_{1}\right) x, \ldots,\left(A_{n}-\lambda_{n}\right) x\right) \quad \text { for } x \in \ell^{2} .
$$

The assumption $\lambda \in \sigma_{\ell e}(A)$ is clearly equivalent to requiring that $T$ not be left invertible modulo the compact operators. Since $\left(\ell^{2}\right)^{n} \simeq \ell^{2}$, standard Fredholm theory implies that either Ker $T$ is infinite-dimensional or $\operatorname{Im} T$ is not closed. In both cases one easily constructs an infinite-dimensional orthogonal projection $P_{1} \in L\left(\ell^{2}\right)$ with the property $T P_{1} \in K\left(\ell^{2},\left(\ell^{2}\right)^{n}\right)$, and hence $\left(A_{i}-\lambda_{i}\right) P_{1} \in K\left(\ell^{2}\right)$ for all $i=1, \ldots, n$. By duality, a similar argument yields that there is an infinitedimensional orthogonal projection $P_{2} \in L\left(\ell^{2}\right)$ with $P_{2}\left(B_{i}-\mu_{i}\right) \in K\left(\ell^{p}\right)$ for $i=1, \ldots, n$. It follows that for all $i=1, \ldots, n$ we have

$$
\left(L_{A_{i}}-\lambda_{i}\right)\left(L_{P_{1}} R_{P_{2}}\right) \in W(I) \quad \text { and } \quad\left(R_{B_{i}}-\mu_{i}\right)\left(L_{P_{1}} R_{P_{2}}\right) \in W(I),
$$

where the operator $L_{P_{1}} R_{P_{2}}$ is not weakly compact on $I$ according to Theorem 3 . Hence the $2 n$-tuple $\left(L_{A}-\lambda, R_{B}-\mu\right)$ fails to be left invertible modulo weakly compact operators, which proves the desired inclusion, and (3.1) follows. The equality (3.2) is proved in a completely symmetric manner, and (3.3) follows from (3.1) and (3.2).

The following corollary is a direct consequence of the spectral mapping theorem for the Harte spectrum.

Corollary 7. Under the assumptions of the previous theorem we have that

$$
\sigma_{H w}\left(P\left(L_{A}, R_{B}\right): I \rightarrow I\right)=P\left(\sigma_{\ell e}(A) \times \sigma_{r e}(B)\right) \cup P\left(\sigma_{r e}(A) \times \sigma_{\ell e}(B)\right)
$$

for polynomials $P: \mathbb{C}^{2 n} \rightarrow \mathbb{C}^{k}(k \in \mathbb{N})$.

In the proof of Theorem 6 above, we used the following fact: If operator $T \in L\left(\ell^{2}\right)$ has closed range and finite-dimensional kernel, then it is left-invertible modulo the compact operators. This fails for operators on $\ell^{p}$ if $p \neq 2$, as is easily shown using the fact ([29], [5]) that these spaces contain uncomplemented copies of themselves, see [3, p. 547] for details. Thus, in order to establish the formula for $\sigma_{w}\left(\mathcal{E}_{A, B}\right)$ in the case of reflexive $\ell^{p}$-spaces $(p \neq 2)$ we must proceed another route. This is the content of the following theorem, which provides a complete extension of [31, Theorem 4(ii)] to elementary operators.

Theorem 8. Let $1<p<\infty$ and assume that $A$ and $B$ are commuting $n$-tuples of operators on $\ell^{p}$. If I stands for a non-reflexive Banach ideal of $L\left(\ell^{p}\right)$ then

$$
\sigma_{w}\left(\mathcal{E}_{A, B}: I \rightarrow I\right)=\sigma_{T e}(A) \circ \sigma_{T e}(B)=\sigma_{H e}(A) \circ \sigma_{H e}(B)
$$


and, more generally,

$$
\begin{aligned}
\sigma_{w}\left(P\left(L_{A}, R_{B}\right): I \rightarrow I\right) & =P\left(\sigma_{T e}(A) \times \sigma_{T e}(B)\right) \\
& =P\left(\sigma_{H e}(A) \times \sigma_{H e}(B)\right)
\end{aligned}
$$

for all polynomials $P: \mathbb{C}^{2 n} \rightarrow \mathbb{C}$.

Proof. Our aim is to prove the inclusions

$$
\begin{aligned}
P\left(\sigma_{a e}(A) \times \sigma_{a e}(B)\right) & \subset \sigma_{w}\left(P\left(L_{A}, R_{B}\right): I \rightarrow I\right) \\
& \subset P\left(\sigma_{H e}(A) \times \sigma_{H e}(B)\right) .
\end{aligned}
$$

Once this is established, (3.7) follows directly from Proposition 1 and clearly (3.6) is a special case of (3.7) corresponding to the choice $P(z, w)=z \circ w$ for $z$, $w \in \mathbb{C}^{n}$.

We start by noting that the spectral mapping theorem yields the right-hand inclusion of (3.8) once we show that $\sigma_{H w}\left(\left(L_{A}, R_{B}\right): I \rightarrow I\right) \subset \sigma_{H e}(A) \times \sigma_{H e}(B)$. But this is a direct consequence of the inclusions (3.4) and (3.5), the proof of which remains valid in the case of the $\ell^{p}$-spaces.

We are left with the left-hand inclusion of (3.8). Suppose to this end that $\lambda \in \sigma_{a e}(A)$ and $\mu \in \sigma_{a e}(B)$. It has to be shown that $P(\lambda, \mu) \in \sigma_{w}\left(P\left(L_{A}, R_{B}\right)\right)$. By applying the Taylor expansion, we write

$$
P(z, w)-P(\lambda, \mu)=G(z, w) \circ(z-\lambda)+H(z, w) \circ(w-\mu), \quad(z, w) \in \mathbb{C}^{n},
$$

with suitably chosen $n$-tuples of polynomials $G=\left(G_{1}, \ldots G_{n}\right)$ and $H=$ $\left(H_{1}, \ldots H_{n}\right)$ (which may depend on $\lambda$ and $\mu$ ). In the rest of the argument we consider the operators on $I$ as elements of $\mathcal{W}(I)=L(I) / W(I)$ via the canonical projection but, for the sake of simplicity, we do not indicate this explicitly. Denote $\Phi:=P\left(L_{A}, R_{B}\right)-P(\lambda, \mu) \in \mathcal{W}(I)$ and assume, contrary to the claim, that $\Phi$ is invertible in the weak Calkin algebra $\mathcal{W}(I)$. We consider separately four alternatives.

$\ggg$ Case 1: $\lambda \in \sigma_{\pi e}(A), \mu \in \sigma_{\delta e}(B)$.

By the assumption, there are operators $U_{k}, V_{k} \in L\left(\ell^{p}\right)$ for $k=1,2, \ldots$ with the properties: $\left\|U_{k}\right\|,\left\|V_{k}\right\| \leq 2,\left\|U_{k}\right\|_{e}=\left\|V_{k}\right\|_{e}=1$ together with $\left\|\left(A_{i}-\lambda_{i}\right) U_{k}\right\|_{e} \rightarrow 0$ and $\left\|V_{k}\left(B_{i}-\mu_{i}\right)\right\|_{e} \rightarrow 0$ for each $i=1, \ldots, n$ as $k \rightarrow \infty$. Write $\Psi_{k}=L_{U_{k}} R_{V_{k}}$ for $k \in \mathbb{N}$. It follows that

$$
\begin{aligned}
\Phi \Psi_{k} & =\sum_{i=1}^{n}\left[G_{i}\left(L_{A}, R_{B}\right)\left(L_{A_{i}}-\lambda_{i}\right)+H_{i}\left(L_{A}, R_{B}\right)\left(R_{B_{i}}-\mu_{i}\right)\right] L_{U_{k}} R_{V_{k}} \\
& =\sum_{i=1}^{n}\left(G_{i}\left(L_{A}, R_{B}\right) L_{\left(A_{i}-\lambda_{i}\right) U_{k}} R_{V_{k}}\right)+\sum_{i=1}^{n}\left(H_{i}\left(L_{A}, R_{B}\right) R_{V_{k}\left(B_{i}-\mu_{i}\right)} L_{U_{k}}\right) .
\end{aligned}
$$


Here $L_{\left(A_{i}-\lambda_{i}\right) U_{k}} \rightarrow 0$ and $R_{V_{k}\left(B_{i}-\mu_{i}\right)} \rightarrow 0$ in $\mathcal{W}(I)$ as $k \rightarrow \infty$ while $L_{U_{k}}$ and $R_{V_{k}}$ stay bounded. Hence $\Phi \Psi_{k} \rightarrow 0$ in $\mathcal{W}(I)$ and this contradicts the assumed invertibility of $\Phi$ since $\left\|\Psi_{k}\right\|_{\mathcal{W}(I)}=\left\|U_{k}\right\|_{e}\left\|V_{k}\right\|_{e}=1$ for all $k \in \mathbb{N}$, according to [31, Theorem 4(i)].

\Case 2: $\lambda \in \sigma_{\delta e}(A), \mu \in \sigma_{\pi e}(B)$.

The argument is completely analogous to Case 1. Now we find elements $\hat{\Psi}_{k}=L_{U_{k}} R_{V_{k}} \in \mathcal{W}(I)$ with the properties that $\left\|\hat{\Psi}_{k}\right\|_{\mathcal{W}(I)}=1$ and $\hat{\Psi}_{k} \Phi \rightarrow 0$ as $k \rightarrow \infty$.

\Case 3: $\lambda \in \sigma_{\pi e}(A), \mu \in \sigma_{\pi e}(B)$.

There are operators $U_{k}, V_{k} \in L\left(\ell^{p}\right)$ for $k=1,2, \ldots$ such that $\left\|U_{k}\right\|,\left\|V_{k}\right\| \leq 2$, $\left\|U_{k}\right\|_{e}=\left\|V_{k}\right\|_{e}=1$ together with $\left\|\left(A_{i}-\lambda_{i}\right) U_{k}\right\|_{e} \rightarrow 0$ and $\left\|\left(B_{i}-\mu_{i}\right) V_{k}\right\|_{e} \rightarrow 0$ for each $i=1, \ldots, n$, as $k \rightarrow \infty$. Denote $\Theta_{k}=\Phi^{-1} L_{U_{k}}$ for $k \in \mathbb{N}$, where the inverse is taken with respect to $\mathcal{W}(I)$. We note that, clearly, $L_{A_{i}-\lambda_{i}}$ and $\Phi$ commute for every $i \in\{1, \ldots, n\}$ and hence, as $k \rightarrow \infty$,

$$
\Phi\left(L_{A_{i}-\lambda_{i}} \Theta_{k}\right)=L_{A_{i}-\lambda_{i}} \Phi \Theta_{k}=L_{\left(A_{i}-\lambda_{i}\right) U_{k}} \rightarrow 0
$$

in $\mathcal{W}(I)$. We deduce that $L_{A_{i}-\lambda_{i}} \Theta_{k} \rightarrow 0$ in $\mathcal{W}(I)$ as $k \rightarrow \infty$ since $\Phi$ is invertible, and this holds for each $i=1, \ldots, n$. It follows that

$$
\begin{aligned}
R_{V_{k}} L_{U_{k}} & =R_{V_{k}} \Phi \Theta_{k} \\
& =\sum_{i=1}^{n} R_{V_{k}} G_{i}\left(L_{A}, R_{B}\right)\left(L_{A_{i}-\lambda_{i}} \Theta_{k}\right)+\sum_{i=1}^{n} R_{\left(B_{i}-\mu_{i}\right) V_{k}} H_{i}\left(L_{A}, R_{B}\right) \Theta_{k}
\end{aligned}
$$

(here one uses the fact that $H_{i}\left(L_{A}, R_{B}\right)$ and $R_{B_{i}-\mu_{i}}$ commute). Evidently, the expression above implies that the element $L_{U_{k}} R_{V_{k}}$ tends to zero in $\mathcal{W}(I)$ as $k \rightarrow \infty$, which again contradicts the fact $\left\|R_{V_{k}} L_{U_{k}}\right\|_{\mathcal{W}(I)}=1$.

$\ggg$ Case 4: $\lambda \in \sigma_{\delta e}(A), \mu \in \sigma_{\delta e}(B)$.

There are suitably normalized $U_{k}, V_{k} \in L\left(\ell^{p}\right)$ such that $\left\|U_{k}\left(A_{i}-\lambda_{i}\right)\right\|_{e} \rightarrow 0$ and $\left\|V_{k}\left(B_{i}-\mu_{i}\right)\right\|_{e} \rightarrow 0$ for each $i=1, \ldots, n$ as $k \rightarrow \infty$. This time we consider $\tilde{\Theta}_{k}=L_{U_{k}} \Phi^{-1}$ and show first that $\tilde{\Theta}_{k} L_{A_{i}-\lambda_{i}} \Phi \rightarrow 0$ and hence that $\tilde{\Theta}_{k} L_{\left(A_{i}-\lambda_{i}\right)} \rightarrow$ 0 for each $i$ as $k \rightarrow \infty$. The contradiction is achieved as in Case 3 by using the equality $L_{U_{k}} R_{V_{k}}=\tilde{\Theta}_{k} \Phi R_{V_{k}}$ to establish that $L_{U_{k}} R_{V_{k}} \rightarrow 0$ in $\mathcal{W}(I)$ as $k$ tends to infinity. This ends the proof of the theorem.

Our next goal will be Theorem 11, which deals with the non-reflexive case. We begin with an auxiliary result which helps us to compute upper estimates for spectra of elementary operators on Banach ideals.

Lemma 9. Let $E$ be a Banach space and assume that $I$ is a Banach ideal of $L(E)$. Then $\sigma_{e}\left(L_{K_{1}} R_{K_{2}}: I \rightarrow I\right)=\{0\}$ whenever $K_{1} \in K(E)$ and $K_{2} \in K(E)$. 
Proof. Assume that $K_{1}, K_{2} \in K(E)$. We first show that

$$
L_{U} R_{K_{2}} \in K(I) \quad \text { for finite dimensional operators } U \in L(E) .
$$

It is enough to consider $U=e^{\prime} \otimes e$ with $e \in E$ and $e^{\prime} \in E^{\prime}$. As in the proof of [31, Theorem 2(i)] we see that $\left(L_{U} R_{K_{2}}\right) B_{I} \subset\left\|e^{\prime}\right\| \Phi\left(K_{2}^{\prime} B_{E^{\prime}}\right)$, where $\Phi \in L\left(E^{\prime}, I\right)$ is defined by $\Phi x^{\prime}=x^{\prime} \otimes e$ for $x^{\prime} \in E^{\prime}$. The claim (3.9) follows since the set $K_{2}^{\prime} B_{E^{\prime}}$ is relatively compact by the assumption that $K_{2} \in K(E)$.

In the general case we assume that $\varepsilon>0$ is given, and we apply the Riesz theory of spectral projections in order to deduce that there is a finite-dimensional $U \in L(E)$ such that the spectral radius of the operator $K_{1}-U$ satisfies $\rho\left(K_{1}-\right.$ $U)<\varepsilon /\left\|K_{2}\right\|$. Since $\left\|\left(L_{K_{1}-U} R_{K_{2}}\right)^{n}\right\|_{L(I)}=\left\|\left(K_{1}-U\right)^{n}\right\|\left\|\left(K_{2}\right)^{n}\right\|$, the spectral radius formula implies that

$$
\rho\left(L_{K_{1}-U} R_{K_{2}}: I \rightarrow I\right)<\varepsilon .
$$

Combined with (3.9), this implies that $\sigma_{e}\left(L_{K_{1}} R_{K_{2}}: I \rightarrow I\right)=\sigma_{e}\left(L_{K_{1}-U} R_{K_{2}}\right.$ : $I \rightarrow I) \subset\{z \in \mathbb{C}|| z \mid \leq \varepsilon\}$, which yields the claim since $\varepsilon>0$ was arbitrary.

Remark. It is a known problem whether the assumptions of the lemma imply that $L_{K_{1}} R_{K_{2}} \in K(I)$. This is at least true if $E$ has the approximation property (use (3.9)), or if $I=L(E)$ (Vala's Theorem, cf. [36, Theorem 3]).

The next proposition provides crucial estimates for joint weak essential spectra of the $2 n$-tuple $\left(L_{A}, R_{B}\right)$. We remark that every Banach ideal $I$ of $L(E)$ is non-reflexive whenever $E$ is non-reflexive, since $E$ embeds isometrically in $I$. Recall that for operator $n$-tuples $B=\left(B_{1}, \ldots, B_{n}\right)$ we denote $B^{\prime}=\left(B_{1}^{\prime}, \ldots, B_{n}^{\prime}\right)$.

Proposition 10. Let $E$ be a non-reflexive Banach space and let I stand for a Banach ideal of $L(E)$. For commuting $n$-tuples $A$ and $B$ of operators on $E$, it holds that:

(1) $\sigma_{H w}\left(\left(L_{A}, L_{B}\right): I \rightarrow I\right) \subset \sigma_{H e}\left(\left(L_{A}, L_{B}\right): I \rightarrow I\right) \subset \sigma_{H e}(A) \times \sigma_{H}(B) \cup$ $\sigma_{H}(A) \times \sigma_{H e}(B)$

(2) $\sigma_{H w}(A) \times \sigma_{a}(B) \cup \sigma_{a}(A) \times \sigma_{H w}\left(B^{\prime}\right) \subset \sigma_{\mathcal{W}(I)}^{\prime \prime}\left(\left(L_{A}, R_{B}\right): I \rightarrow I\right)$,

where $\sigma_{\mathcal{W}(I)}^{\prime \prime}$ denotes the joint bicommutant spectrum in the algebra $\mathcal{W}(I)$.

Proof.

(i) It is certainly enough to establish the right-hand inclusion. We shall combine Lemma 9 with an argument from [4]. Since the computation is short, we include the details. Denote $\overline{0}:=(0, \ldots, 0) \in \mathbb{C}^{n}$. By the translation invariance of the joint spectra, it is enough to show that the assumption

$$
(\overline{0}, \overline{0}) \notin \sigma_{H e}(A) \times \sigma_{H}(B) \cup \sigma_{H}(A) \times \sigma_{H e}(B)
$$


implies that $(\overline{0}, \overline{0}) \notin \sigma_{H e}\left(\left(L_{A}, R_{B}\right): I \rightarrow I\right)$. If $\overline{0} \notin \sigma_{H}(A)$, then there are $n$-tuples $S^{1}$ and $S^{2}$ on $E$ with the property $A \circ S^{1}=S^{2} \circ A=\operatorname{Id}_{E}$ so that $\left(L_{S^{2}}, 0\right) \circ\left(L_{A}, R_{B}\right)=\left(L_{A}, R_{B}\right) \circ\left(L_{S^{1}}, 0\right)=\operatorname{Id}_{I}$ and hence $(\overline{0}, \overline{0}) \notin \sigma_{H e}\left(\left(L_{A}, R_{B}\right)\right.$ : $I \rightarrow I)$. The argument is similar if $\overline{0} \notin \sigma_{H}(B)$. The remaining possibility is that $\overline{0} \notin \sigma_{H e}(A) \cup \sigma_{H e}(B)$. In this case there are $n$-tuples $S^{(i)}$ on $E$ and compact operators $K_{i} \in K(E)$ for $i=1,2,3,4$, such that

$$
S^{(1)} \circ A+K_{1}=A \circ S^{(2)}+K_{2}=S^{(3)} \circ B+K_{3}=B \circ S^{(4)}+K_{4}=\operatorname{Id}_{E} .
$$

A computation reveals that

$$
\begin{aligned}
& \left(L_{S^{(1)}}, R_{S^{(4)}} L_{K_{1}}\right) \circ\left(L_{A}, R_{B}\right)+L_{K_{1}} R_{K_{4}}=\operatorname{Id}_{I}=\left(L_{A}, R_{B}\right) \circ\left(L_{S^{(2)}}, R_{S^{(3)}} L_{K_{2}}\right) \\
& +L_{K_{2}} R_{K_{3}} \text {, }
\end{aligned}
$$

where products of type $R_{S^{(2)}} L_{K_{1}}$ are to be understood as $R_{S^{(4)}} L_{K_{1}}=$ $\left(R_{S_{1}^{(4)}} L_{K_{1}}, \ldots, R_{S_{n}^{(4)}} L_{K_{1}}\right)$ if $S^{(4)}=\left(S_{1}^{(4)}, \ldots, S_{n}^{(4)}\right)$. Here the operators $\operatorname{Id}_{I}-$ $L_{K_{1}} R_{K_{4}}$ and $\operatorname{Id}_{I}-L_{K_{2}} R_{K_{3}}$ are Fredholm according to Lemma 9, and the claim follows as we multiply from left and right by the corresponding Fredholm inverses.

(ii) We assume that $(\overline{0}, \overline{0}) \notin \sigma_{\mathcal{W}(I)}^{\prime \prime}\left(\left(L_{A}, R_{B}\right): I \rightarrow I\right)$. Hence there is an operator $2 n$-tuple $\Psi=\left(\Gamma_{1}, \ldots, \Gamma_{n}, \Phi_{1}, \ldots, \Phi_{n}\right) \in(L(I))^{2 n}$ and a weakly compact operator $\mathcal{V} \in W(I)$ such that the elements of the set

$$
\left\{L_{A_{1}}, \ldots, L_{A_{n}}, R_{B_{1}}, \ldots R_{B_{n}}, \Gamma_{1}, \ldots, \Gamma_{n}, \Phi_{1}, \ldots, \Phi_{n}\right\}
$$

commute modulo $W(I)$, and such that

$$
\Psi \circ\left(L_{A}, R_{B}\right)+\mathcal{V}=\operatorname{Id}_{I}=\left(L_{A}, R_{B}\right) \circ \Psi+\mathcal{V} .
$$

Our strategy consists in showing that the assumption $\overline{0} \in \sigma_{a}(B)$ (resp. $\overline{0} \in$ $\left.\sigma_{a}(A)\right)$ implies that $\overline{0} \notin \sigma_{H w}(A)$ (resp. $\overline{0} \notin \sigma_{H w}\left(B^{\prime}\right)$ ). Accordingly, the proof is divided into two parts.

$\ggg$ Case 1: $\overline{0} \in \sigma_{a}(B)$.

There are bounded sequences $\left(x_{k}^{\prime}\right)$ in $E^{\prime}$ and $\left(x_{k}\right)$ in $E$ such that either $B_{i} x_{k} \rightarrow 0$ for all $i=1, \ldots, n$ as $k \rightarrow \infty$, or $B_{i}^{\prime} x_{k}^{\prime} \rightarrow 0$ for all $i=1, \ldots, n$ as $k \rightarrow \infty$, and with the property that $\left\langle x_{k}^{\prime}, x_{k}\right\rangle=1$ for all $k \in \mathbb{N}$. For indices $i \in\{1, \ldots, n\}$ and $k \in \mathbb{N}$, we define the operators $S_{k}^{i}, R_{k}, V_{k}$ on $E$ by the formulas

$$
\begin{gathered}
S_{k}^{i} x=\left(\Gamma_{i}\left(x_{k}^{\prime} \otimes x\right)\right) x_{k}, \\
R_{k} x=\sum_{i=1}^{n}\left(\Phi_{i}\left(x_{k}^{\prime} \otimes x\right)\right) B_{i} x_{k}
\end{gathered}
$$


and

$$
V_{k} x=\left(\mathcal{V}\left(x_{k}^{\prime} \otimes x\right)\right) x_{k}
$$

for arbitrary $x \in E$. All these operators are clearly linear and bounded (note that the canonical embedding $I \rightarrow L(E)$ is contractive by definition). Moreover, $V_{k}$ is weakly compact since it may be written as a product of operators, one of which is weakly compact. We claim that for all $k \in \mathbb{N}$, it holds that

$$
\sum_{i=1}^{n} A_{i} S_{k}^{i}+R_{k}+V_{k}=\operatorname{Id}_{E}
$$

and that

$$
A_{i} S_{k}^{i}-S_{k}^{i} A_{i} \in W(E) \quad(i=1, \ldots, n)
$$

Before verifying (3.15) - (3.17), we indicate how the desired conclusion $\overline{0} \notin$ $\sigma_{H w}(A)$ is obtained if one assumes their validity. Namely, once $k$ is chosen large enough so that $\left\|R_{k}\right\|_{w}<1$, we may use the Neumann series to invert $\operatorname{Id}_{E}-R_{k}$ $\bmod W(E)$. Then (3.15) implies that $\sum_{i=1}^{n} A_{i} S_{k}^{i}$ is a weak Fredholm operator, and hence $\overline{0} \notin \sigma_{r w}(A)$. Using (3.16), we similarly obtain that $\overline{0} \notin \sigma_{\ell w}(A)$ and hence that $\overline{0} \notin \sigma_{H w}(A)$.

It remains to establish (3.15)-(3.17). We start from the equality $\left(L_{A}, R_{B}\right) \circ$ $\Psi+\mathcal{V}=\operatorname{Id}_{I}$, and evaluate both sides at $x_{k}^{\prime} \otimes x \in I$ (here $x \in E$ is arbitrary), with the result

$$
\sum_{i=1}^{n} A_{i} \Gamma_{i}\left(x_{k}^{\prime} \otimes x\right)+\sum_{i=1}^{n} \Phi_{i}\left(x_{k}^{\prime} \otimes x\right) B_{i}+\mathcal{V}\left(x_{k}^{\prime} \otimes x\right)=x_{k}^{\prime} \otimes x .
$$

This operator identity is next evaluated at the point $x_{k}$, which yields (3.15).

In order to prove (3.16), we note first that $\Gamma_{i}$ and $L_{A_{i}}$ commute mod $W(I)$ for each $i=1, \ldots, n$ so that $L_{A_{i}} \Gamma_{i}-\Gamma_{i} L_{A_{i}}=\mathcal{V}_{i}$ for some $\mathcal{V}_{i} \in W(I)$. Evaluating this first at $x_{k}^{\prime} \otimes x$ and thereafter at $x_{k}$, we see that

$$
A_{i} S_{k}^{i} x-\left(\Gamma_{i}\left(x_{k}^{\prime} \otimes A_{i} x\right)\right) x_{k}=\left(\mathcal{V}_{i}\left(x_{k}^{\prime} \otimes x\right)\right) x_{k} .
$$

The message is clear: $A_{i} S_{k}^{i}-S_{k}^{i} A_{i} \in W(E)$.

Finally, in the case that $B_{i} x_{k} \rightarrow 0$ as $k \rightarrow \infty, i \in\{1, \ldots, n\}$, we obtain that $\left\|R_{k}\right\| \leq \sum_{i=1}^{n}\left\|\Phi_{i}\right\|\left\|x_{k}^{\prime}\right\|\left\|B_{i} x_{k}\right\| \rightarrow 0$ as $k \rightarrow \infty$, and this implies (3.17). Assume next that the second possibility holds: $B_{i}^{\prime} x_{k}^{\prime} \rightarrow 0$ as $k \rightarrow \infty, i \in\{1, \ldots, n\}$. Since $R_{B_{i}}$ and $\Phi_{i}$ commute mod $W(I)$, there are $\mathcal{U}_{i} \in W(I)$ such that $R_{B_{i}} \Phi_{i}-\Phi_{i} R_{B_{i}}=$ $\mathcal{U}_{i}$. The already familiar evaluation yields that

$$
R_{k} x=\sum_{i=1}^{n}\left(\Phi_{i}\left(B_{i}^{\prime} x_{k}^{\prime} \otimes x\right)\right) x_{k}+\sum_{i=1}^{n}\left(\mathcal{U}_{i}\left(x_{k}^{\prime} \otimes x\right)\right) x_{k} .
$$


This evidently implies (3.17), and the proof of Case 1 is complete.

$\ggg$ Case 2. $\overline{0} \in \sigma_{a}(A)$.

Again there are bounded sequences $\left(x_{k}^{\prime}\right)$ in $E^{\prime}$ and $\left(x_{k}\right)$ in $E$ such that $\left\langle x_{k}^{\prime}, x_{k}\right\rangle=$ 1 for all $k \in \mathbb{N}$ and either $A_{i} x_{k} \rightarrow 0(i=1, \ldots, n)$ as $k \rightarrow \infty$, or $A_{i}^{\prime} x_{k}^{\prime} \rightarrow 0$ $(i=1, \ldots, n)$ as $k \rightarrow \infty$. The operators $\tilde{S}_{k}^{i}, \tilde{R}_{k}, \tilde{V}_{k}$ on $E^{\prime}$, for $i \in\{1, \ldots, n\}$ and $k \in \mathbb{N}$, are defined as follows:

$$
\begin{gathered}
\tilde{S}_{k}^{i} x^{\prime}=\left(\Phi_{i}\left(x^{\prime} \otimes x_{k}\right)\right)^{\prime} x_{k}^{\prime}, \\
\tilde{R}_{k} x^{\prime}=\sum_{i=1}^{n}\left(\Gamma_{i}\left(x^{\prime} \otimes x_{k}\right)\right)^{\prime} A_{i}^{\prime} x_{k}^{\prime}
\end{gathered}
$$

and

$$
\tilde{V}_{k} x^{\prime}=\left(\mathcal{V}\left(x^{\prime} \otimes x_{k}\right)\right)^{\prime} x_{k}^{\prime},
$$

for arbitrary $x^{\prime} \in E^{\prime}$. All the operators defined above are bounded since they may be written as compositions of bounded linear operators (note that the adjoint operation: $T \mapsto T^{\prime}$ is an isometric embedding $\left.L(E) \rightarrow L\left(E^{\prime}\right)\right)$. We have

$$
\begin{array}{ll}
\sum_{i=1}^{n} B_{i}^{\prime} \tilde{S}_{k}^{i}+\tilde{R}_{k}+\tilde{V}_{k}=\operatorname{Id}_{E^{\prime}}, & \\
B_{i}^{\prime} \tilde{S}_{k}^{i}-\tilde{S}_{k}^{i} B_{i}^{\prime} \in W\left(E^{\prime}\right) & (i=1, \ldots, n)
\end{array}
$$

and

$$
\left\|\tilde{R}_{k}\right\|_{w} \rightarrow 0 \text { as } k \rightarrow \infty .
$$

Assuming the validity of $(3.21)-(3.23)$, we deduce that $\overline{0} \notin \sigma_{H w}\left(B^{\prime}\right)$ exactly as in Case 1. The equality (3.21) is obtained by evaluating the identity $\left(L_{A}, R_{B}\right) \circ$ $\Psi+\mathcal{V}=\operatorname{Id}_{I}$ first at $x^{\prime} \otimes x_{k}$ with arbitrary $x^{\prime} \in E^{\prime}$, taking adjoints, and finally evaluating the resulting identity at $x_{k}^{\prime}$. Equalities (3.22) and (3.23) are similarly proved, following the outline of Case 1, and we may safely leave their proof to the reader.

We are ready to establish one of the main results of the paper. Recall that the class of Banach spaces possessing the DPP contains all $\mathcal{L}^{1}$ - and $\mathcal{L}^{\infty}$-spaces, among which we mention $c_{0}, \ell^{1}, \ell^{\infty}, C(0,1), L^{1}(0,1), L^{\infty}(0,1)$ and all their dual spaces-see [22, II.4.30].

Theorem 11. Let $E$ be an infinite-dimensional Banach space such that $E^{\prime}$ has the Dunford-Pettis property and let I stand for a Banach ideal of $L(E)$. Then for commuting operator $n$-tuples $A$ and $B$ on $E$, one has that

$$
\begin{aligned}
\sigma_{w}\left(\mathcal{E}_{A, B}: I \rightarrow I\right) & =\sigma_{T e}(A) \circ \sigma_{T}(B) \cup \sigma_{T}(A) \circ \sigma_{T e}(B) \\
& =\sigma_{H e}(A) \circ \sigma_{H}(B) \cup \sigma_{H}(A) \circ \sigma_{H e}(B)
\end{aligned}
$$


and, more generally,

$$
\begin{aligned}
\sigma_{w}\left(P\left(L_{A}, R_{B}\right): I \rightarrow I\right) & =P\left(\sigma_{T e}(A) \times \sigma_{T}(B)\right) \cup P\left(\sigma_{T}(A) \times \sigma_{T e}(B)\right) \\
& =P\left(\sigma_{H e}(A) \times \sigma_{H}(B)\right) \cup P\left(\sigma_{H}(A) \times \sigma_{H e}(B)\right)
\end{aligned}
$$

for all polynomials $P: \mathbb{C}^{2 n} \rightarrow \mathbb{C}$.

Proof. The spectral mapping theorem applied to Proposition 10(ii) yields that

$$
P\left(\sigma_{H w}(A) \times \sigma_{a}(B)\right) \cup P\left(\sigma_{a}(A) \times \sigma_{H w}\left(B^{\prime}\right)\right) \subset \sigma_{w}\left(P\left(L_{A}, R_{B}\right)\right) .
$$

Since $E^{\prime}$ has the DPP, $E$ has the DPP too (this follows directly from the definition of the DPP), and hence we may replace in (3.24) the spectra $\sigma_{H w}$ by $\sigma_{H e}$. Namely, assume for instance that $(A-\lambda) \circ S=\operatorname{Id}_{E}+V$, where $S=\left(S_{1}, \ldots, S_{n}\right)$ and $V \in W(E)$. It follows that $(A-\lambda) \circ \tilde{S}=\operatorname{Id}_{E}-V^{2}$ with $\tilde{S}_{i}=S_{i}\left(\operatorname{Id}_{E}-V\right)$ for $i=1, \ldots, n$, and here $V^{2} \in K(E)$ by the Dunford-Pettis property of $E$.

In conclusion, Proposition 1 implies that there is the inclusion

$$
P\left(\sigma_{H e}(A) \times \sigma_{H}(B)\right) \cup P\left(\sigma_{H}(A) \times \sigma_{H e}(B)\right) \subset \sigma_{w}\left(P\left(L_{A}, R_{B}\right)\right),
$$

and Proposition 10(i) in combination with the spectral mapping theorem turns this into an equality. Finally, we may replace the Harte spectra by the Taylor spectra according to Proposition 1.

Remark. We point out that our proof easily modifies to yield the formula

$$
\sigma_{e}\left(P\left(L_{A}, R_{B}\right): I \rightarrow I\right)=P\left(\sigma_{T e}(A) \times \sigma_{T}(B)\right) \cup P\left(\sigma_{T}(A) \times \sigma_{T e}(B)\right),
$$

due to Eschmeier [12, Corollary 3.3] (Eschmeier proves this formula also for analytic $P$ ). In fact, this is obtained simply by dropping the assumption on $E^{\prime}$ and replacing the weak essential spectra by the essential spectra and the weakly compact operators by the compact operators in the proofs of Proposition 10 and Theorem 11. In addition, the formula

$$
\sigma\left(P\left(L_{A}, R_{B}\right): I \rightarrow I\right)=P\left(\sigma_{T}(A) \times \sigma_{T}(B)\right)
$$

can be proved by similar means.

In [31] an example is given of a space $E$ such that $E^{\prime}$ has the DPP but $L(E)$ fails to have the DPP. This excludes the possibility that in general the equality $\sigma_{w}\left(\mathcal{E}_{A, B}: L(E) \rightarrow L(E)\right)=\sigma_{e}\left(\mathcal{E}_{A, B}: L(E) \rightarrow L(E)\right)$ provided by Theorem 11 could be obtained by establishing the DPP for $L(E)$. 
4. Distance formulas. A map $D \in L\left(L\left(\ell^{2}\right)\right)$ is called a derivation if it satisfies $D(U V)=U D(V)+D(U) V$ for all $U, V \in L\left(\ell^{2}\right)$. It is well known (for a proof, see $\left[20\right.$, p. 228]) that every derivation $D$ on $L\left(\ell^{2}\right)$ is inner, that is, $D=D_{T}:=L_{T}-R_{T}$ for some $T \in L\left(\ell^{2}\right)$. In this section we determine the weak essential norm of an inner derivation on $L\left(\ell^{2}\right)$ and the essential norm of the two-sided multiplication operator $L_{A} R_{B}$ on $L\left(\ell^{2}\right)$, where $A, B \in L\left(\ell^{2}\right)$.

The following theorem complements the well-known Stampfli identity [33, Theorem 4] stating that $\left\|D_{T}\right\|=2 \inf _{\lambda \in \mathbb{C}}\|T-\lambda\|$ and the formula for the essential norm $\left\|D_{T}\right\|_{e}=\inf _{\lambda \in \mathbb{C}}\left(\|T-\lambda\|+\|T-\lambda\|_{e}\right)$, due to C. K. Sun ([34]).

Theorem 12. Let $T \in L\left(\ell^{2}\right)$. Then

$$
\left\|D_{T}: L\left(\ell^{2}\right) \rightarrow L\left(\ell^{2}\right)\right\|_{w}=2 \inf _{\lambda \in \mathbb{C}}\|T-\lambda\|_{e} .
$$

Proof. Since $\left\|L_{T}\right\|_{w}=\left\|R_{T}\right\|_{w}=\|T\|_{e}$ by [31, Theorem 2(ii)], we see that $\left\|D_{T}\right\|_{w}=\left\|L_{T-\lambda}-R_{T-\lambda}\right\|_{w} \leq 2\|T-\lambda\|_{e}$ for all $\lambda \in \mathbb{C}$. It remains to prove the converse inequality. Without loss of generality, it is enough to show that $\left\|D_{T}\right\|_{w} \geq 2$ assuming that $1=\|T\|_{e} \leq\|T+\lambda\|_{e}$ for all $\lambda \in \mathbb{C}$. The assumptions combined with [16, Propositions 2.1 and 3.2] provide an infinite-dimensional orthogonal projection $P \in L\left(\ell^{2}\right)$ with the properties

$$
\|T P\|_{e}=1 \quad \text { and } \quad P T P:=K_{1} \in K\left(\ell^{2}\right) .
$$

Let $\varepsilon>0$. Standard basic sequence techniques (compare with the proof of $\left[31\right.$, Lemma 1]) and the assumption $\|T P\|_{e}=\left\|T P-K_{1}\right\|_{e}=1$ produce a (closed) infinite-dimensional subspace $M_{1} \subset \operatorname{Im}(P)$ such that the operator $\left(T P-K_{1}\right)_{\mid M_{1}}$ is an $(1+\varepsilon)$-isomorphism onto the image $M_{2}:=\left(T P-K_{1}\right) M_{1}$. That is,

$$
(1+\varepsilon)^{-1}\|x\| \leq\left\|\left(T P-K_{1}\right) x\right\| \leq(1+\varepsilon)\|x\|
$$

for $x \in M_{1}$. Let $Q_{1}$ (resp. $Q_{2}$ ) denote the orthogonal projection onto $M_{1}$ (resp. onto $\left.M_{2}\right)$. Note that $\left.\left(T P-K_{1}\right)\right|_{M_{1}}=\left.\left(T Q_{1}-K_{1}\right)\right|_{M_{1}}$. The identity $P T P=K_{1}$ implies that $P K_{1}=K_{1}$, and hence we obtain for $x, y \in M_{1}$ that

$$
\left\langle\left(T P-K_{1}\right) x, y\right\rangle=\left\langle\left(T P-K_{1}\right) x, P y\right\rangle=\left\langle\left(P T P-P K_{1}\right) x, y\right\rangle=0 .
$$

Thus $M_{1} \perp M_{2}$ or, in other words, $Q_{1} Q_{2}=Q_{2} Q_{1}=0$.

Let $S \in L\left(\ell^{2}\right)$ satisfy

$$
\left\{\begin{array}{l}
S\left(T P-K_{1}\right) Q_{1}=Q_{1} \\
S\left(1-Q_{2}\right)=0 .
\end{array}\right.
$$

Actually $\left.S\right|_{M_{2}}=\left(\left.\left(T P-K_{1}\right)\right|_{M_{1}}\right)^{-1}$ so that $\|S\|<1+\varepsilon$. In addition, we see that $\left(T P-K_{1}\right) S Q_{2}=Q_{2}$ and $S=S Q_{2}=Q_{1} S=Q_{1} S Q_{2}$. 
Define $\Omega \in L\left(L\left(\ell^{2}\right)\right)$ by setting $\Omega=L_{Q_{2}} R_{S}+L_{S} R_{Q_{1}}$. We claim that

$$
\|\Omega\| \leq 1+\varepsilon .
$$

Namely, let $x \in B_{\ell^{2}}$ and $U \in B_{L\left(\ell^{2}\right)}$. We obtain $\Omega(U) x=Q_{2} U S x+S U Q_{1} x=$ $Q_{2} U S Q_{2} x+Q_{1} S U Q_{1} x$. Using the observations $\left\langle Q_{2} U S Q_{2} x, Q_{1} S U Q_{1} x\right\rangle=0$ and $\left\|Q_{1} x\right\|^{2}+\left\|Q_{2} x\right\|^{2} \leq 1$ together with the fact $\|S\| \leq 1+\varepsilon$, we deduce that $\|\Omega(U) x\| \leq 1+\varepsilon$ and (4.2) follows.

Define $\Phi \in L\left(L\left(\ell^{2}\right)\right)$ by $\Phi=L_{T} R_{Q_{1}}+R_{T Q_{1}}$. Then

$$
R_{Q_{1}} D_{T} R_{\left(2 Q_{1}-\mathrm{Id}\right)}=\Phi-2 R_{Q_{1} T Q_{1}},
$$

where $\left\|R_{Q_{1}}\right\|=\left\|R_{2 Q_{1}-\mathrm{Id}}\right\|=1$ and $R_{Q_{1} T Q_{1}}=R_{Q_{1} P T P Q_{1}}=R_{Q_{1} K_{1} Q_{1}} \in$ $W\left(L\left(\ell^{2}\right)\right)$. Hence, it suffices to prove that

$$
\|\Phi\|_{w} \geq 2 .
$$

To this end we note that

$$
\Phi \Omega=L_{T Q_{2}} R_{S Q_{1}}+L_{Q_{2}} R_{S T Q_{1}}+L_{T S} R_{Q_{1}}+L_{S} R_{Q_{1} T Q_{1}} .
$$

Here $L_{S} R_{Q_{1} T Q_{1}} \in W\left(L\left(\ell^{2}\right)\right)$ and $L_{T Q_{2}} R_{S Q_{1}}=0$ since $S Q_{1}=S Q_{2} Q_{1}=0$. Moreover, (4.1) implies that $S T Q_{1}=Q_{1} \bmod K\left(\ell^{2}\right)$ and (as above) $T S=$ $T P S Q_{2}=Q_{2} \bmod K\left(\ell^{2}\right)$. We end up with the result

$$
\Phi \Omega=2 L_{Q_{2}} R_{Q_{1}}+\mathcal{V}
$$

where $\mathcal{V} \in W\left(L\left(\ell^{2}\right)\right)$ and $\left\|L_{Q_{2}} R_{Q_{1}}\right\|_{w}=\left\|Q_{2}\right\|_{e}\left\|Q_{1}\right\|_{e}=1$ by [31, Theorem 2(ii)]. Thus (4.3) follows from (4.2) and (4.4) since $\varepsilon>0$ was arbitrary.

Remark. Let $I$ stand for a non-reflexive Banach ideal of $L\left(\ell^{2}\right)$. With minor modifications, the proof above yields the estimates

$$
\left(\frac{2}{\|\Omega\|_{L(I)}}\right) \inf _{\lambda \in \mathbf{C}}\|T-\lambda\|_{e} \leq\left\|D_{T}: I \rightarrow I\right\|_{w} \leq 2 \inf _{\lambda \in \mathbf{C}}\|T-\lambda\|_{e},
$$

where $\Omega$ is defined as in the course of the proof of Theorem 12 above. We remark that Fialkow [13] showed that the Stampfli identity does not hold for restrictions on certain Banach ideals of $L\left(\ell^{2}\right)$. Moreover, Johnson [19] constructed twodimensional operators $T$ on $\ell^{p}(1<p<\infty, p \neq 2)$ such that $\| D_{T}: L\left(\ell^{p}\right) \rightarrow$ $L\left(\ell^{p}\right)\left\|<2 \inf _{\lambda \in \mathbb{C}}\right\| T-\lambda \|$.

Our last theorem complements the formula $\left\|L_{A} R_{B}: I \rightarrow I\right\|_{w}=\|A\|_{e}\|B\|_{e}$ ([31, Theorem 2(ii)]), where $A, B \in L\left(\ell^{2}\right)$ and $I$ is a Banach ideal of $L\left(\ell^{2}\right)$. It should be noted, that trivially $\left\|L_{A} R_{B}: I \rightarrow I\right\|=\|A\|\|B\|$. 
Theorem 13. Let $A, B \in L\left(\ell^{2}\right)$. Then

$$
\left\|L_{A} R_{B}: I \rightarrow I\right\|_{e}=\max \left\{\|A\|\|B\|_{e},\|A\|_{e}\|B\|\right\}
$$

for any Banach ideal I of $L\left(\ell^{2}\right)$.

Proof. The proof of the lower estimate

$$
\left\|L_{A} R_{B}: I \rightarrow I\right\|_{e} \geq \max \left\{\|A\|\|B\|_{e},\|A\|_{e}\|B\|\right\}
$$

is completely analogous to that of [31, Theorem 5(i)] - it should also be remembered that $\|B\|_{e}=\left\|B^{\prime}\right\|_{e}$ for $B \in L\left(\ell^{2}\right)$. Towards the converse inequality, we note first that if $A \in K\left(\ell^{2}\right)$ or $B \in K\left(\ell^{2}\right)$, the claim easily follows. For instance, if $A$ is compact, we write $B=B_{1}+K_{1}$ with $\left\|B_{1}\right\| \leq\|B\|_{e}+\varepsilon$ and $K_{1} \in K\left(\ell^{2}\right)$ for given $\varepsilon>0$. The decomposition $L_{A} R_{B}=L_{A} R_{B_{1}}+L_{A} R_{K_{1}}$ yields that $\left\|L_{A} R_{B}: I \rightarrow I\right\|_{e} \leq\left\|L_{A} R_{B_{1}}\right\|=\|A\|\left(\|B\|_{e}+\varepsilon\right)$, while $L_{A} R_{K_{1}} \in K(I)$ since it is clearly approximable in norm by finite-dimensional operators.

Assume then that neither $A$ nor $B$ is compact. By scaling, we may assume that $\|A\|_{e}=1=\|B\|_{e}$. Let $d=\max \{\|A\|,\|B\|\}$. Using the polar decomposition of $A^{*}$ and $B$, write $A=T_{1} U_{1}$ and $B=U_{2} T_{2}$, where $U_{i}$ are partial isometries and $T_{i}$ are positive operators for $i=1,2$. The properties of the polar decomposition readily imply that $\left\|T_{i}\right\|_{e}=1$ and $\left\|T_{i}\right\| \leq d(i=1,2)$. It is enough to verify that

$$
\left\|L_{T_{1}} R_{T_{2}}: I \rightarrow I\right\|_{e} \leq d,
$$

since $L_{A} R_{B}=\left(L_{T_{1}} R_{T_{2}}\right)\left(L_{U_{1}} R_{U_{2}}\right)$ and $\left\|L_{U_{1}} R_{U_{2}}\right\| \leq 1$. We may also assume that $\left\|T_{1}\right\|>1$ and $\left\|T_{2}\right\|>1$, since otherwise $\left\|L_{T_{1}} R_{T_{2}}\right\|=d$.

Suppose next that $\varepsilon>0$ is given. We apply the spectral resolution of the positive operator $T_{1}$. Let $P_{1}$ be the spectral projection corresponding to $\sigma\left(T_{1}\right) \cap$ ] $1+\varepsilon, \infty\left[\right.$. The fact that $\left\|T_{1}\right\|>1=\|T\|_{e}$ implies that $P_{1}$ is a finite dimensional orthogonal projection, non-zero for small enough $\varepsilon$, such that $T_{1}$ admits an operator matrix of type $\left(\begin{array}{cc}H_{1} & 0 \\ 0 & K_{1}\end{array}\right)$ with respect to the orthogonal decomposition $\ell^{2}=X_{1} \oplus Y_{1}$, where $X_{1}=\left(1-P_{1}\right) \ell^{2}$ and $Y_{1}=P_{1} \ell^{2}$. Here $\left\|H_{1}\right\| \leq 1+\varepsilon$ and $\left\|K_{1}\right\| \leq d$. In a similar manner, we deduce that there are a finite-dimensional projection $P_{2}$, subspaces $X_{2}=\left(1-P_{2}\right) \ell^{2}$ and $Y_{2}=P_{2} \ell^{2}$, and operators $H_{2}, K_{2}$ such that $\left\|H_{2}\right\| \leq 1+\varepsilon, \quad\left\|K_{2}\right\| \leq d$, and $T_{2}=\left(\begin{array}{cc}H_{2} & 0 \\ 0 & K_{2}\end{array}\right)$ with respect to the decomposition $\ell^{2}=X_{2} \oplus Y_{2}$.

Define $\Phi \in L(I)$ by $\Phi=L_{T_{1}} R_{T_{2}}+L_{P_{1}} R_{P_{2}}\left(\operatorname{Id}_{I}-L_{T_{1}} R_{T_{2}}\right)$. We claim that

$$
\|\Phi\| \leq(1+\varepsilon)^{2} d .
$$

This is sufficient to establish (4.5), since $L_{P_{1}} R_{P_{2}} \in K(I)$ (note that the operator $L_{P_{1}} R_{P_{2}}$ is finite-dimensional) and $\varepsilon$ may be chosen arbitrary small. 
Assume that $U \in I$ satisfies $\|U\|_{I}=1$ and admits the operator matrix

$$
U=\left(\begin{array}{ll}
U_{11} & U_{12} \\
U_{21} & U_{22}
\end{array}\right): X_{2} \oplus Y_{2} \rightarrow X_{1} \oplus Y_{1} .
$$

A direct computation shows that

$$
\Phi(U)=\left(\begin{array}{cc}
H_{1} U_{11} H_{2} & H_{1} U_{12} K_{2} \\
K_{1} U_{21} H_{2} & U_{22}
\end{array}\right): X_{2} \oplus Y_{2} \rightarrow X_{1} \oplus Y_{1} .
$$

In order to estimate $\|\Phi(U)\|_{I}$, we first note that

$$
\Phi(U)=\left(\begin{array}{cc}
H_{1} & 0 \\
0 & \operatorname{Id}_{Y_{1}}
\end{array}\right)\left(\begin{array}{cc}
U_{11} & U_{12} K_{2} \\
K_{1} U_{21} & U_{22}
\end{array}\right)\left(\begin{array}{cc}
H_{2} & 0 \\
0 & \operatorname{Id}_{Y_{2}}
\end{array}\right),
$$

where $\left\|\left(\begin{array}{cc}H_{i} & 0 \\ 0 & \operatorname{Id}_{Y_{i}}\end{array}\right)\right\| \leq 1+\varepsilon$ for $i=1,2$. Moreover,

$$
\left(\begin{array}{cc}
U_{11} & U_{12} K_{2} \\
K_{1} U_{21} & U_{22}
\end{array}\right)=\left(\begin{array}{cc}
U_{11} & U_{12} \\
U_{21} & U_{22}
\end{array}\right)+\left(\begin{array}{cc}
0 & U_{12}\left(K_{2}-\operatorname{Id}_{Y_{2}}\right) \\
\left(K_{1}-\operatorname{Id}_{Y_{1}}\right) U_{21} & 0
\end{array}\right) .
$$

By combining the previous observations, we see that (4.6) follows, once we show that $\left\|\left(\begin{array}{cc}0 & U_{12} S_{2} \\ S_{1} U_{21} & 0\end{array}\right)\right\|_{I} \leq d-1$, where $S_{i}=K_{i}-\operatorname{Id}_{Y_{i}}(i=1,2)$. To this end, we observe that the decomposition

$$
\begin{aligned}
\left(\begin{array}{cc}
0 & U_{12} \\
U_{21} & 0
\end{array}\right)= & \frac{1}{2}\left(\begin{array}{ll}
U_{11} & U_{12} \\
U_{21} & U_{22}
\end{array}\right) \\
& +\frac{1}{2}\left(\begin{array}{cc}
-\operatorname{Id}_{X_{1}} & 0 \\
0 & \operatorname{Id}_{Y_{1}}
\end{array}\right)\left(\begin{array}{ll}
U_{11} & U_{12} \\
U_{21} & U_{22}
\end{array}\right)\left(\begin{array}{cc}
\operatorname{Id}_{X_{2}} & 0 \\
0 & -\operatorname{Id}_{Y_{2}}
\end{array}\right)
\end{aligned}
$$

yields $\left\|\left(\begin{array}{cc}0 & U_{12} \\ U_{21} & 0\end{array}\right)\right\|_{I} \leq 1$. Moreover, the definition of the operators $K_{i}$ implies that $\operatorname{Id}_{Y_{i}} \leq K_{i} \leq d \operatorname{Id}_{Y_{i}}$ so that $\left\|S_{i}\right\| \leq d-1$, for $i=1,2$. Hence,

$$
\begin{aligned}
\left\|\left(\begin{array}{cc}
0 & U_{12} S_{2} \\
S_{1} U_{21} & 0
\end{array}\right)\right\|_{I}= & (d-1) \|\left(\begin{array}{cc}
\operatorname{Id}_{X_{1}} & 0 \\
0 & (d-1)^{-1} S_{1}
\end{array}\right) \\
& \times\left(\begin{array}{cc}
0 & U_{12} \\
U_{21} & 0
\end{array}\right)\left(\begin{array}{cc}
\operatorname{Id}_{X_{2}} & 0 \\
0 & (d-1)^{-1} S_{2}
\end{array}\right) \|_{I} \\
\leq & d-1
\end{aligned}
$$

and the theorem follows.

Problem. It is probable that the distance formula of Theorem 13 is not correct if $\ell^{2}$ is replaced by an arbitrary Banach space $E$ (the corresponding formula does not hold in general for tensor products of operators - see [2, Example 7.7]). However, we ask whether Theorem 13 remains valid when $E=\ell^{p}$ with $1<p<\infty, p \neq 2$. 


\section{REFERENCES}

[1] C. Akemann \& S. Wright: Compact actions on $C^{*}$-algebras, Glasgow Math. J. 21 (1980), 143-149.

[2] K. Astala: On measures of noncompactness and ideal variations on Banach spaces, Ann. Acad. Sci. Fenn. Ser. AI Math. Diss. 29 (1980).

[3] K. Astala 85 H-O. Tylli: On semi-Fredholm operators and the Calkin algebra, J. London Math. Soc. 34 (1986), 541-551.

[4] A. Carrillo 8 C. Hernández: Spectra of constructs of a system of operators, Proc. Amer. Math. Soc. 91 (1981), 426-432.

[5] G. Bennett, L. Dor, V. Goodman, W.B. Johnson \& C. M. Newman: On uncomplemented subspaces of $L^{p}(1<p<2)$, Israel J. Math. 26 (1977) 178-187.

[6] R. E. Curto: The spectra of elementary operators, Indiana Univ. Math. J. 32 (1983) 193-197.

[7] R. E. Curto: Connections between Harte and Taylor spectra, Rev. Roum. Math. Pures Appl. 32 (1986), 203-215.

[8] R. E. Curto: Applications of several complex variables to multiparameter spectral theory. In : J. Conway and B. Morrel (eds.): Surveys of Some Recent Results in Operator Theory, vol. II, pp. 25-90. Pitman Research Notes in Math. Ser. 192. Longman 1988.

[9] R. E. CurTo: Spectral theory of elementary operators. In: Mathieu M. (ed.): Elementary Operators and Applications, pp. 3-52. World Scientific 1992

[10] R. E. Curto 8 L. A. Fialkow: The spectral picture of $\left[L_{A}, R_{B}\right]$, J. Funct. Anal. 71 (1987), 371-392.

[11] J. Diestel 83 B. Faires: Remarks on the classical Banach operator ideals, Proc. Amer. Math. Soc. 58 (1976), 189-196.

[12] J. EschmeIER: Tensor products and elementary operators., J. Reine Angew. Math. 390 (1988), 47-66.

[13] L. A. Fialkow: $A$ note on norm ideals and the operator $X \mapsto A X-X B$, Israel J. Math. 32 (1979). 331-348.

[14] L. A. Fialkow: Essential spectra of elementary operators, Trans. Amer. Math. Soc. 267 (1981), 157-174.

[15] L. A. Fialkow: Structural properties of elementary operators. In: M. Mathieu (ed.): Elementary Operators and Applications, pp. 55-113. World Scientific 1992.

[16] C. K. Fong: On the essential maximal numerical range, Acta Sci. Math. 41 (1979), 307-315.

[17] C.K. Fong $\&$ A.R. Sourour: On the operator identity $\sum A_{k} X B_{k}=0$, Canad. J. Math. 31 (1979), 845-857.

[18] R. Harte: Spectral mapping theorems, Proc. Royal Irish Acad. 72A (1972), 89-107.

[19] B. E. Johnson: Norms of derivations on L(X), Pacific J. Math 38 (1971), 465-469.

[20] B. E. Johnson: Characterization and norms of derivations on von Neumann algebras. In: Pierre de la Harpe (ed.): Algebres d'Operateurs, Lecture Notes in Math. 725, pp. 228-235. Springer 1979.

[21] A. Lebow \& M. Schechter: Semigroups of operators and measures of noncompactness, J. Funct. Anal. 7 (1971), 1-26.

[22] J. Lindenstrauss 86 L.Tzafriri: Classical Banach Spaces. Lecture Notes in Math. vol. 338. Springer 1973.

[23] J. Lindenstrauss 83 L.Tzafriri: Classical Banach Spaces I. Sequence Spaces. Ergebnisse der Mathematik vol. 92. Springer 1977.

[24] B. Magajna: A system of operator equations, Canad. Math. Bull. 30 (1987), 200-209.

[25] B. Magajna: A transitivity theorem for algebras of elementary operators, Proc. Amer. Math. Soc. 118 (1993), 119-127.

[26] M. Mathieu: Elementary operators on prime $C^{*}$-algebras, II, Glasgow Math. J. 30 (1988), 275-284.

[27] T. Ogasawara: Finite-dimensionality of certain Banach algebras, J. Sci. Hiroshima Univ. Ser. A17 (1954), 359-364. 
[28] G. RACHER: On the tensor product of weakly compact operators, Math. Ann. 294 (1992), 267-275.

[29] H. P. Rosenthal: On the subspaces of $L^{p}(p>2)$ spanned by sequences of independent random variables, Israel J. Math 8 (1970), 273-303.

[30] E. Saksman 86 H.-O. Tylli: Weak compactness of multiplication operators on spaces of bounded linear operators, Math. Scand. 70 (1992), 91-111.

[31] E. Saksman $\&$ H.-O. Tylli: Weak essential spectra of multiplication operators on spaces of bounded linear operators, Math. Ann. 299 (1994), 299-309.

[32] Z. Slodkowski 63 W. Zelazko: On joint spectra of commuting families of operators, Studia Math. 50 (1974), 127-148.

[33] J. G. Stampfli: The norm of a derivation, Pacific J. Math. 33 (1970), 737-747.

[34] C. K. Sun: The essential norm of generalized derivation operators, Chinese Ann. Math. Ser. A13 (1992) no. 2, 211-221 (Chinese).

[35] J. L. TAYLOR: A joint spectrum for several commuting operators, J. Funct. Anal. 6 (1970), 172-191.

[36] K. Vala: On compact sets of compact operators, Ann. Acad. Sci. Fenn. A I Math. 351 (1964).

[37] K. Ylinen: Weakly completely continuous elements of $C^{*}$-algebras, Proc. Amer. Math. Soc. 52 (1975), 323-326.

The author gratefully acknowledges support by the Academy of Finland.

Department of Mathematics

P. O. Box 4 (Hallituskatu 15)

$\mathrm{SF}-00014$ University of Helsinki

Finland

E-mail: saksman@cc.helsinki.fi

Received: August 1st, 1994. 